(CAuteur(es). Cette œuvre, disponible à

http://dx.doi.org/10.18162/fp.2020.520, est distribuée sous licence Creative Commons Attribution 4.0 International http://creativecommons.org/licences/by/4.0/deed.fr

Chantal Ouellet

Université du Québec à Montréal (Canada)

Amal Boultif

Université d'Ottawa (Canada)

Annie Dubeau

Université du Québec à Montréal (Canada)

\section{Le journal de bord comme outil d'accompagnement d'enseignants en formation professionnelle intégrant une approche favorisant la compréhension en lecture}

\author{
The logbook as a support tool for vocational training \\ teachers integrating a reading comprehension approach
}

doi: 10.18162/fp.2020.520
Les enseignants en formation professionnelle du secondaire (FP) s'attendent à ce que les élèves possèdent les habiletés de lecture nécessaires pour lire les contenus des cours, mais bon nombre d'entre eux éprouvent des difficultés. C'est ainsi qu'un total de 21 enseignants de la FP a été accompagné dans l'implantation d'une approche pédagogique probante en compréhension en lecture sur deux années consécutives.

L'analyse qualitative de leurs journaux de bord révèle que les modalités d'appropriation de l'approche expérimentée

passent par des bonifications et des adaptations, un modelage plus fréquent de stratégies de lecture de haut

niveau, une plus grande variété de regroupements des élèves lors de la lecture collaborative et une forte présence de la pratique du désétayage progressif.

\section{Mots-clés}

Lecture, formation professionnelle, accompagnement, journal de bord.

Abstract

Secondary VET teachers expect students to have the reading skills necessary to read course content, but many are struggling. Thus, 21 VET teachers were accompanied in the implementation of a proven pedagogical approach in reading comprehension. The qualitative analysis of their logbooks reveals that the methods of appropriating the approach go through improvements and adaptations, a more frequent modeling of high-level reading strategies, a greater variety of groupings of students during collaborative reading and a strong presence of the practice of gradual release of responsibility.

Keywords Reading, vocational training, logbook.

\section{Problématique}

La capacité de traiter l'information écrite dans des environnements lettrés, numériques ou non, constitue un enjeu fondamental pour assurer l'autonomie des travailleurs et leur capacité à se former tout au long de leur vie. Or, les résultats québécois du Programme pour l'évaluation internationale des compétences des adultes (PEICA) (Statistique Canada, 2013) mettent en évidence les faibles niveaux de littératie d'une partie importante de la population âgée de 16 à 65 ans, $49 \%$ se situant aux niveaux 1 et 2 . Précisons que les niveaux 1 (capacités de lecture très faibles) et 2 (capacités à traiter des textes simples) correspondent à l'analphabétisme fonctionnel. Parmi les établissements d'enseignement où se retrouvent des adolescents et des adultes ayant un faible niveau de littératie, les centres de formation professionnelle (FP) du secondaire accueillent une population diversifiée d'élèves qui ont connu des parcours scolaires souvent difficiles (Hamelin, 2014; Tremblay, 2010) et qui néprouvent pas d'intérêt pour les études de plus longue haleine, comme les études collégiales et universitaires (Deschenaux, 2007; Mazalon et Bourdon, 2015).

Or, les FP sont exigeantes et les élèves ne s'attendent pas nécessairement à ce qu'elles soient si difficiles. Ainsi, l'achèvement des programmes de FP est ardu pour une importante proportion d'élèves comme en témoigne l'étude de Dubeau, Plante et Frenay (2017). Cette recherche a permis de documenter que 64,5\% des élèves ayant pris part à l'étude ont éprouvé des difficultés pendant leur parcours de FP. De ce nombre, 13,5 \% ont abandonné leur formation et $51 \%$ des élèves persévérants présentaient des difficultés sur le plan scolaire. Trois principales raisons peuvent expliquer les difficultés de ces élèves et soutiennent l'importance de développer leur capacité à 
traiter l'information écrite dans des environnements lettrés et numériques pendant leur parcours de FP. D'abord, en contexte montréalais, un grand nombre de personnes nées hors Canada fréquentent un centre de FP et sont allophones (29,6 \%). De plus, plusieurs centres accueillent des élèves dont la lecture en français n'est pas fluide (Pouliot, 2015). De plus, la majorité des effectifs de la FP du secondaire du Québec sont regroupés dans la filière menant au diplôme d'études professionnelles (DEP) qui compte plus de 140 programmes (Gouvernement du Québec, 2019). Les préalables minimalement requis en français, en anglais et en mathématiques varient selon le programme convoité et les diverses conditions prévues par le Régime pédagogique de la formation professionnelle (Gouvernement du Québec, 2017). Ce faisant, les compétences initiales en français sont variables d'un élève à l'autre à l'amorce d'une FP : certains disposeront des unités en français de la $5^{\mathrm{e}}$ secondaire, d'autres de celles de la $4^{\mathrm{e}}$ ou de la $3^{\mathrm{e}}$ secondaire. Enfin, la FP est offerte selon deux principales modalités d'enseignement. La première, l'approche d'enseignement traditionnelle, comporte des cours magistraux donnés en groupe. La deuxième modalité,l'approche individualisée, comporte des activités théoriques et pratiques qui s'effectuent individuellement, permettant aux élèves de cheminer à leur propre rythme dans leur formation sur la base de matériel didactique écrit (Misiorowska, 2014). Même si l'écrit occupe une place centrale dans l'approche d'enseignement individualisée, du matériel didactique écrit est également utilisé par les enseignants dans l'approche traditionnelle. De plus, dans certains programmes de FP, la formation offerte de façon traditionnelle ou individualisée se fait presque entièrement à l'ordinateur (p. ex., dans les DEP de secrétariat et de comptabilité). Ce faisant, les formations qui en découlent comportent de plus en plus de lectures complexes sur des supports imprimés et numériques. Or, en classe, plusieurs élèves ont un niveau insuffisant en lecture pour comprendre de façon autonome les contenus des cours à apprendre pendant leur FP (Grossmann, Roiné et Chatigny, 2014), que la lecture se fasse sur support papier ou numérique. Devant ces constats, il est peu surprenant que ces élèves éprouvent des difficultés scolaires pour terminer leur FP (Dubeau et al., 2017; Mazalon et Bourdon, 2015).

Même si l'enseignement de stratégies de lecture dans différentes disciplines scolaires gagne du terrain (Shanahan et Shanahan, 2008), l'enseignement de stratégies de compréhension de lecture relève habituellement de la spécialité des enseignants de français et nécessite d’y être bien formé, ce qui n'est pas le cas des enseignants de la FP du secondaire qui n'y sont pas formés dans le cadre de leur formation universitaire à l'enseignement (voir par exemple la présentation du programme de baccalauréat en enseignement professionnel de l'UQAM et de l'Université de Sherbrooke). En même temps, la compréhension en lecture est intimement liée au domaine dans lequel on lit. Ainsi, les élèves devraient développer graduellement leur habileté à lire, à écrire et à réfléchir selon les modalités qui caractérisent les disciplines scolaires (p. ex., logique du discours, structures de textes, vocabulaire spécialisé). Ce sont alors les enseignants spécialistes, et lecteurs experts du domaine, qui sont les mieux placés pour enseigner à mieux comprendre les textes dans leur propre domaine (p. ex, lire à la façon d'un historien, d'un comptable, etc.) (Buehl, 2011; Schoenbach et Greenleaf, 2017). Bien que de nouveaux modèles éducatifs en formation générale du secondaire préconisent l'enseignement de la compréhension en lecture dans différentes disciplines, de façon intégrée, par les enseignants spécialistes de contenu, il n'existe au Canada ni ailleurs (p. ex., Nouvelle-Zélande, Royaume-Uni) aucun modèle d'intégration complète de stratégies de lecture à l'enseignement d'un métier, par des enseignants de FP eux-mêmes, sur lequel nous pouvions nous appuyer. Dans un tel modèle, l'intégration complète prendrait alors 
forme dans l'enseignement simultané de stratégies de lecture et de l'enseignement spécifique à une discipline, en l'occurrence ici d'un métier, par le même enseignant, soit l'enseignant de secrétariat, de coiffure, etc.

Notre équipe de trois co-chercheuses et de deux assistantes de recherche s'est alors tournée vers l'approche Reading Apprenticeship (RA) (Schoenbach et Greenleaf, 2017; Schoenbach, Greenleaf et Murphy, 2012) comme modèle d'intégration de stratégies de lecture afin de tenter de répondre aux besoins d'un centre de formation professionnelle les ayant inscrites dans son plan de réussite et qui nous a sollicité pour lui venir en aide. L'approche RA préconise la prise de conscience de ses propres stratégies de lecture et leur bonification selon les exigences des textes. Cette approche renferme également des routines d'enseignement, décrites plus loin, qui permettent aux lecteurs "novices », au regard de la lecture dans une discipline donnée, de développer leur maitrise de la compréhension au moyen de stratégies plus efficaces. Dans le cadre d'un projet de recherche-action-formation, nous nous sommes donc appuyés sur cette approche pour former des enseignants de FP et les accompagner lors de la planification de leurs cours et de leur enseignement, entre autres, à toutes les quinzaines au moyen de journaux de bord. Nous nous sommes aussi donné pour objectif de documenter, d'une part, comment ce groupe d'enseignants qui forment à un métier pouvait s'approprier une telle approche permettant d'enseigner des stratégies de lecture au moyen de routines à leurs élèves et, d'autre part, les retombées de cette approche dans le cadre de leur enseignement.

Le présent article vise à décrire : 1) l'intégration déclarée, dans le journal de bord des enseignants, des stratégies de compréhension en lecture dans les cours de FP ainsi que leur enseignement inspiré de l'approche RA et 2) les retombées perçues du recours à ces stratégies pour l'enseignement d'un métier.

\section{Cadre de référence}

La compréhension en lecture est un concept très vaste ayant donné lieu à l'élaboration de nombreux modèles théoriques. Depuis une quinzaine d'années, elle est abordée, dans bon nombre d'écoles secondaires québécoises, sous l'angle des "stratégies de lecture » souvent présentes dans les plans de réussite. L'approche Reading Apprenticeship (Schoenbach et Greenleaf, 2017; Schoenbach et al., 2012), dont nous nous sommes inspirés, va au-delà de l'enseignement de stratégies de lecture. Elle accorde une grande importance à l'engagement de l'enseignant dans la découverte de ses propres processus et stratégies de compréhension lorsqu'il lit dans sa discipline, ce qu'il est amené à modéliser ou à rendre visible auprès de ses élèves qui, à leur tour, auront des occasions de lire en classe de manière « active » et soutenue.

\section{Une approche pédagogique qui favorise la compréhension en lecture dans différentes disciplines}

L'approche RA, dont les fondements comportent des aspects socioconstructivistes (lecture collaborative), socioculturels (apports de différents textes et des forces des étudiants) et cognitivistes (lecture stratégique), permet d'outiller les étudiants en compréhension en lecture dans des cours portant sur la langue d'enseignement (p. ex., le français ou l'anglais), mais aussi dans les différentes disciplines composant leur programme d'études. Cette approche convenait au contexte particulier des enseignants 
et des élèves du centre de formation professionnelle du secondaire qui nous a sollicités étant donné qu'elle a été développée en contexte d'enseignement au secondaire avec des adolescents et des jeunes adultes.

Pour Schoenbach et al. (2012), l'approche RA intègre quatre dimensions. Premièrement, la dimension personnelle permet à lélève de se donner du pouvoir par rapport à sa lecture et de développer sa confiance en soi et son autonomie, de devenir conscient de ses forces et de ses faiblesses en lecture et de se fixer des buts. Deuxièmement, la dimension sociale consiste à s'approprier les stratégies efficaces des pairs ou de l'enseignant qui agit comme modèle lors des interactions ou discussions sur un texte présentant des difficultés et à les partager. Troisièmement, la dimension cognitive favorise l'accès aux stratégies de lecture que le lecteur expert utilise dans son domaine, soit l'enseignant, de même que les bons lecteurs de façon générale, afin de résoudre des problèmes de compréhension de textes se situant dans leur zone proximale de développement (ZPD) (Vygotsky, 1978). Avant, pendant et après la lecture du texte, le bon lecteur a recours à différentes stratégies, telles que visualiser le contenu ou identifier les idées principales, qui lui permettent de comprendre le texte. Même s'il existe plus d'une douzaine de stratégies de lecture reconnues efficaces dans les écrits scientifiques, nous avons choisi d'en prioriser cinq : 1) se poser des questions sur le sens d'un passage ou du texte; 2) clarifier les mots de vocabulaire ou les concepts incompris; 3 ) faire des prédictions sur le sens du texte, les infirmer ou les confirmer; 4) résumer un passage ou une portion de texte et 5) faire des liens avec ses connaissances antérieures, du monde ou de la discipline. Toute autre stratégie utile à la compréhension d'un texte est bien sûr prise en compte selon les exigences du texte. Quatrièmement, une dimension relative à la construction des connaissances permet, quant à elle, d'identifier les particularités des différents textes disciplinaires comme le vocabulaire spécifique, les structures de texte, la logique du discours et permet de s'approprier la façon dont un lecteur expert lit et comprend dans sa discipline (p. ex., lire comme un philosophe, comme un historien) et, dans le cas qui nous préoccupe, lire comme un technicien en comptabilité, une secrétaire médicale, etc. (Shanahan et Shanahan, 2008). Toutes ces dimensions sont chapeautées par la discussion métacognitive interne et externe du lecteur. La discussion métacognitive interne consiste à faire une réflexion à voix haute qui sera éventuellement intériorisée par le lecteur. Cette réflexion à voix haute intériorisée se transpose à l'écrit au moyen du " parler au texte » (talk to the text) qui consiste à annoter ou, autrement dit, à laisser des traces écrites sur le texte en fonction de ses propres réflexions, en interaction avec le texte, et lors de l'application de stratégies. La discussion métacognitive externe réfère à la construction du sens réalisée lors des interactions et des discussions en petits groupes et en grand groupe (Schoenbach et al., 2012).

Ces différentes dimensions de l'approche RA sont intégrées par l'enseignant et les élèves lors de routines en classe. Typiquement, la routine débute par : 1) un modelage par l'enseignant d'une portion de texte où ce dernier rend visibles les stratégies qu'il utilise au moyen de la réflexion à haute voix; 2) la lecture d'une autre portion de texte par les élèves, individuellement; 3) un partage, en petits groupes, des difficultés de compréhension du texte et des stratégies utilisées; 4) un partage, en grand groupe, des solutions trouvées en petits groupes pour arriver à comprendre le texte; 5) de façon autonome, la rédaction d'une réflexion individuelle sur l'activité de compréhension du texte ou la réalisation de toute tâche désignée par l'enseignant s'appuyant sur la compréhension du texte lu comme la lecture d'une mise en situation à la suite de laquelle une tâche en comptabilité est effectuée. Cette routine permet un désétayage progressif (gradual release of responsibility) où l'enseignant diminue graduellement son 
soutien aux élèves. Bien que ces composantes de la routine soient présentées ici en ordre, elles peuvent tout aussi bien être exécutées dans un ordre différent, l’important étant qu'elles soient toutes présentes dans une situation d'enseignement (Fisher et Frey, 2013; Meirieu, s. d.).

Toutefois, l'intégration en classe de stratégies de lecture et de cette routine par des enseignants qui œuvrent dans le domaine de la FP demeure à la fois nouvelle et méconnue. Pour combler ce manque, nous avons documenté l'intégration de l'approche, déclarée par les enseignants formés aux stratégies de compréhension de lecture, de même que les retombées de cet enseignement, tel qu'ils l'ont perçu.

\section{Méthode}

Nos résultats sont issus d'une recherche-action-formation qui accentue le processus de la formation dans la recherche (Prud'homme, 2007; Prud'homme, Dolbec et Guay, 2011). L'enseignement de stratégies de compréhension en lecture étant peu connu des enseignants de FP, nous avons recruté 27 enseignants dans quatre programmes différents : diplôme d'études professionnelles (DEP) en comptabilité, en secrétariat, en soutien informatique et attestation de spécialisation professionnelle (ASP) en secrétariat médical, qui ont été formés à l'approche RA. Au cœur de la recherche-actionformation se trouve l'idée du développement de la science dans et par l'action (Argyris, 1980; SaintArnaud, 2001, 2004; Schön, 1983, 1987), dans laquelle la théorie et la pratique sont intimement liées. Nous visions à remédier à la faiblesse des compétences en compréhension de textes en formation professionnelle, telle qu'identifiée par le milieu, et aux lacunes quant à cette pédagogie du côté des enseignants.

\section{Collecte de données au moyen d'un journal de bord}

Dans cette recherche, nous avons adopté le journal réflexif (Bouchard, 2011; Boud, 2001; Perrenoud, 2001; Schön, 1983, 1987). Lobjectif de ce journal était d'engager les enseignants dans un processus de réflexion et d'analyse d'une situation d'enseignement et d'apprentissage et de documenter l'intégration de l'approche RA à leur pratique (Bouchard, 2011). Par ailleurs, ce type de journal permet le développement de la réflexion sur la pratique, comme une mise à distance de l'action (Saint-Arnaud, 2001), ce qui a des retombées sur les actions futures de l'enseignant (Schön, 1987; Vacher, 2011). Il nous a également servi d'instrument de suivi d'implantation de l'approche et de collecte de données. Chaque enseignant devait envoyer son journal par courriel à un membre de l'équipe de recherche qui lui répondait et lui fournissait une rétroaction par écrit. Selon Boutin (2006), le journal permet au participant de sélectionner les informations qu'il souhaite partager avec le lecteur. Il laisse donc le temps au scripteur de penser au type d'information qu'il souhaite transmettre, dans le cas présent, aux membres de l'équipe de recherche. Le journal de bord des participants est constitué de traces écrites, laissées par l'enseignant participant, dont le contenu concerne ses réflexions, ses questions, des commentaires ou le résumé de ses apprentissages (Derobertmasure, Dehon et Demeuse, 2010; SaintArnaud, 2001, 2004; Schön, 1987; Vacher, 2011), en l'occurrence dans cette recherche, ceux liés à l'enseignement selon l'approche RA (Schoenbach et al., 2012). Seules les traces écrites des enseignants ont fait l'objet de l'analyse, sans la rétroaction du membre de l'équipe de recherche qui avait pour rôle d'y réagir et de les commenter. Afin de faciliter l'analyse des données, un canevas de base a été fourni aux participants comportant une partie descriptive et une partie réflexive (Vacher, 2011). 
Cette série de journaux de bord a été recueillie entre septembre 2015 et janvier 2016. Les enseignants devaient envoyer leur journal aux quinzaines, ce qui nous donne 44 entrées de journaux de bord pour 21 enseignants, les 27 n'ayant pas tous participé à la collecte. Chaque enseignant a partagé entre 2 et 10 entrées de son journal de bord pendant cette période. Les enseignants devaient compléter les portions descriptives et réflexives à partir d'un cours de leur choix et devaient y noter la date, le programme, le module, le titre de la leçon, les modalités de travail, l'activité menée ainsi que les bonifications apportées à leur intégration de la RA. Ils devaient aussi signaler les stratégies les plus sollicitées, décrire le déroulement de l'activité de lecture impliquant un recours à ces stratégies par eux (p. ex., en phase de modelage) ou par les élèves. Dans la portion réflexive, ils devaient faire un retour critique sur l'activité de compréhension en lecture, telle qu'ils l'avaient menée. Les enseignants ont été préalablement formés à l'utilisation du journal de bord par un membre de l'équipe de recherche lors d'une rencontre de suivi en septembre 2015.

\section{Analyses}

Nous avons procédé à une analyse de fréquence des contenus des entrées des journaux de bord (Creswell, 2012) avec codage ouvert (p. ex., stratégies). Puis, pour analyser les descriptions et les réflexions des enseignants sur leurs pratiques d'intégration des stratégies de compréhension en lecture et leur enseignement, nous en avons fait une analyse avec codage ouvert par théorisation ancrée (Paillé et Mucchielli, 2012). Les catégories émergentes, qui sont parvenues à saturation à la suite de l'analyse du tiers environ du corpus, ont été organisées selon qu'elles concernaient les stratégies enseignées, les modalités pédagogiques privilégiées pour les enseigner et les réflexions des enseignants quant aux bonifications apportées. Un contre-codage a été effectué par un autre membre de l'équipe de recherche permettant d'obtenir un accord interjuge de 0,55 et un indice de Kappa de 77,74 \%, soit de modéré à bon.

\section{Présentation et interprétation des résultats}

Dans ce qui suit, nous présentons les résultats de l'analyse des journaux de bord portant sur les aspects descriptifs de la pratique des enseignants, soit l'usage des stratégies de lecture et leur enseignement suivis des objets de leurs réflexions.

\section{L'usage de stratégies de haut niveau}

Dix enseignants sur les 21 ont recours plus systématiquement aux stratégies de lecture et plus spécifiquement aux stratégies dites de haut niveau comme clarifier, résumer,faire des liens ( $\mathrm{n}=29$ entrées). Selon leurs besoins et en fonction de la difficulté des contenus à lire, certains enseignants choisissent de mettre l'accent sur une ou deux stratégies. C'est le critère de pertinence de la ou des stratégies par rapport à l'activité qui les pousse à en faire usage : «je fais un retour sur toutes les stratégies de lecture, mais mets l'accent sur la stratégie "clarifier" lors de la lecture des consignes"; et parfois à en privilégier plus d'une : «c'est principalement les stratégies "se questionner, clarifier et faire des liens" » qui ont été utilisées.

Sans surprise, on note que ce sont les stratégies « clarifier, faire des liens et résumer » qui reviennent le plus souvent dans les journaux des enseignants. C'est le cas pour cet autre enseignant qui note : «J'ai décidé ce mois-ci de sortir de ma zone de confort. [...] J'ai donc choisi les stratégies "faire des liens, clarifier et 
résumer" dans un cours de recherche d'emploi en comptabilité pour un exercice qui était à priori incompris » et qui rajoute, dans une autre entrée de son journal : "Je fais un retour sur toutes les stratégies de lecture, mais j'ai mis l'accent sur la stratégie "résumer" ".

\section{Importance de la clarification du vocabulaire}

Pour les enseignants de la formation professionnelle, la clarification du vocabulaire technique est d'une importance capitale, car c'est de la compréhension de ce dernier que découle la réussite des élèves. La moitié des enseignants déclarent y avoir recours.

Cette fois, je suis allée plus loin dans la définition des termes. Je voulais que les élèves réalisent que le compte banque, celui qui contient l'argent de l'entreprise, se trouve à la banque (l'institution financière). La banque émet la liste des transactions quelle a reçues pendant le mois et qui ont affecté le compte de banque (le relevé bancaire). De son côté, l'entreprise inscrit les transactions qu'elle a faites durant le mois dans ses livres ou registres comptables (système comptable: journal général, journal des ventes, journal des achats, etc.).

Sept enseignants procèdent plus systématiquement à la clarification du vocabulaire spécifique lié aux matières enseignées ( $n=13$ entrées) et cela se fait souvent dans un contexte collaboratif. Et, avec les stratégies de clarification du vocabulaire, la sollicitation des connaissances antérieures et la mise en relation des connaissances au moyen de la stratégie "faire des liens » sont aussi très prisées par les enseignants quand il s'agit d'amorcer de nouveaux contenus avec une nouvelle terminologie ou de rattacher des contenus nouveaux à des cours préalables.

\section{Rappels fréquents des stratégies}

Certains enseignants ( $\mathrm{n}=4,4$ entrées) n'hésitent pas à faire des retours sur les stratégies et leur usage, car ils estiment que cela contribue à aider leurs élèves, mais aussi eux-mêmes, puisque cela leur permet de mieux évaluer les effets de leur pratique sur ces derniers. Cette enseignante en fait le constat :

Même les meilleurs élèves ont appris de nouvelles choses. [...] Ils ont réussi à fournir un travail et à prendre conscience de leur problème de lecture! Ils ont aussi mis en pratique le surlignage, le soulignage, les annotations, le questionnement, la prédiction, la clarification et le résumé pour la plupart. Ils se sont rendu compte que leur problème ne résidait pas toujours dans la manipulation du logiciel, mais dans la comprébension des directives [...].

\section{Lecture et annotation}

L'annotation est une stratégie très sollicitée qui fait maintenant partie des façons de faire de certains enseignants ( $\mathrm{n}=5,8$ entrées). L'annotation sur les documents donne ainsi accès, d'une façon plus précise, aux pannes de compréhension, ce qui permet aux enseignants d'intervenir de façon adaptée et efficace (Schoenbach et al., 2012). Un peu plus de la moitié des enseignants ( $n=12,13$ entrées) font aussi le constat que les élèves ne relisent plus sans avoir d'objectif précis, ils semblent être capables, grâce $\mathrm{au}$ «parler au texte » d'identifier les pannes de compréhension et de cibler les parties non comprises. L'annotation rend leur relecture plus efficace. 


\section{Importance du modelage de l'enseignant}

L'importance du modelage revient souvent dans les journaux de bord et plus spécifiquement chez sept enseignants (12 entrées). C'est surtout lors des interactions avec les étudiants que les enseignants font des rappels et modélisent l'usage des stratégies de lecture les plus appropriées qui répondent aux obstacles rencontrés. Ils associent les stratégies et le modelage de ces dernières pour en tirer un maximum d'effet en les associant aux stratégies d'enseignement efficaces comme pour un enseignant qui note :

Durant l'explication de la nouvelle matière, nous avons produit un chiffrier ensemble en affichant sur un tableau interactif un exercice à compléter. Je complétais le chiffrier en même temps qu'eux tout en leur faisant analyser et en les amenant à faire des liens avec leur formation antérieure.

L'enseignement explicite des stratégies de lecture selon des modalités d'enseignement collaboratives de la lecture sur supports papier et numérique est aussi mis de l'avant par bon nombre d'enseignants. En voici un exemple qui illustre la parfaite intégration des aspects multimodaux de la lecture dans certains cours qui s'appuient sur les supports de lecture papier, mais aussi numériques en ligne et hors ligne :

\section{[...] présentation des stratégies "prédire, clarifier et faire des liens». Demande expresse de survoler dans un premier temps le texte avec l'intention de prédire le contenu du texte de l'évaluation. Par la suite, formation de dyades pour une lecture, toujours à l'écran avec comme mandat de tisser des liens avec ce qui a été appris en classe et de clarifier ce qui est demandé.}

\section{Les réflexions des enseignants mènent à l'adaptation de l'enseignement aux besoins des étudiants}

Des enseignants ( $n=6,9$ entrées) ont aussi recours aux stratégies de lecture lors d'examens ou de formatifs et se servent du questionnement et du modelage pour mieux aider les élèves et dissiper les angoisses de ces derniers. L'approche et leur réflexion sur celle-ci permettent de mieux cibler les difficultés des élèves et d'adapter les pratiques d'enseignement (Saint-Arnaud, 2001) dans le but de cibler les difficultés mentionnées lors des échanges et du questionnement des élèves. On note aussi un réajustement de la pratique (Schön, 1983), par exemple, dans les façons d'enseigner les contenus et une différenciation, grâce aux informations fournies par les annotations des étudiants et par leurs questions :

J'ai fait des liens avec la matière vue en classe, j'ai clarifié certains mots [...] ils ont annoté les questions pour les rendre plus claires, dans leurs mots. Ensuite, j'ai demandé à trois élèves, un pour chacune des questions, et en ciblant des élèves que je savais plus en difficulté, de résumer, dans leur mot, la question demandée. À la fin, tous savaient quoi faire et personne n'est revenu me voir pour me demander des clarifications.

Des enseignants ( $\mathrm{n}=8,10$ entrées) indiquent avoir fréquemment recours à la réflexion métacognitive (interne et externe) et varient davantage les modalités d'organisation durant le travail de lecture. Dans la partie réflexive du journal, à la suite de la description de l'action, les enseignants soulignent le phénomène d'autonomisation des étudiants et un recours plus spontané aux stratégies de lecture :

J'ai remarqué que certains étudiants soulignent les mots importants ou les exigences du travail seuls sans que je l'exige. On voit plus facilement l'autonomie des étudiants avec ce genre de travail. Les stratégies de lecture permettent d'acquérir une certaine autonomie et peuvent aider à développer cette qualité. 


\section{Le désétayage progressif lors de la pratique guidée}

Les descriptions des pratiques d'enseignement en lien avec la RA laissent transparaître une appropriation du désétayage progressif chez 15 enseignants ( $n=21$ entrées). Quels que soient les composantes de la routine (Fisher et Frey, 2013) adoptées pour le permettre (grand groupe, dyades, magistral collaboratif, individualisé, modelage) et l'ordre dans lequel elles sont appliquées, on retrouve dans toutes les séances décrites par les enseignants la verbalisation des pannes de compréhension et le questionnement actif en contexte collaboratif permettant une appropriation graduelle des stratégies par les élèves les rendant de plus en plus autonomes. Trois enseignantes s'adaptent aux besoins des élèves et les incitent à lire de façon individuelle et collaborative pour comparer leurs compréhensions mutuelles des textes et mieux s'approprier les stratégies, sans toujours compter sur elle ou sur un dictionnaire. Ce que ces enseignantes notent dans leurs journaux de bord montre comment elles ont su tirer profit de la dimension sociale et de la collaboration entre pairs pour mieux autonomiser les élèves lors de leurs lectures.

Autonomisation des élèves et importance de la routine de lecture collaborative permettant le désétayage progressif

Il est possible de retracer, dans les journaux de bord, les moments où le désétayage a lieu ou encore, les composantes nécessaires à l'autonomisation des élèves en lecture. L'extrait du journal de bord de cette enseignante est éloquent.

Je demande à une élève de lire à haute voix l'exercice (la mise en situation). Ensuite, chaque élève doit relire la mise en situation individuellement et appliquer les stratégies de lecture. Elles soulignent les moments pertinents, font des liens entre le texte et les composantes de la lettre d'affaires quielles devront rédiger à la suite de l'analyse de l'exercice, et adaptent les phrases de la mise en situation au texte de la lettre tel qu'il va apparaitre en version finale. Après le travail individuel, je forme des dyades. Le but de cette activité est de donner la possibilité aux élèves d'échanger leurs opinions, de poser des questions à leurs pairs, de clarifier avec ses pairs les difficultés et trouver des réponses appropriées. Cette interaction leur permet d'être moins anxieuses, de percevoir les choses d'un autre point de vue, d'apprendre une analyse de texte différente de la sienne.

Certains enseignants ( $n=6,7$ entrées) portent aussi une attention accrue aux aspects métacognitifs liés aux processus d'apprentissage et d'autorégulation chez leurs élèves et se soucient de les faire émerger et de les rendre conscients chez leurs élèves : "Quand je remplis le bordereau de dépôt au tableau, je pose des questions aux élèves sur la façon dont elles ont réussi à placer les bons éléments dans les bonnes cases.»

\section{Importance des retours réflexifs permis par le journal de bord}

La portion réflexive du journal permet de constater que les formations et l'accompagnement offerts par l'équipe de recherche-action, les nombreux échanges par courriels avec les chercheuses au moyen et à la suite de la tenue des journaux de bord permettent l'émergence d'une réflexion sur l'action et sur le geste pédagogique (Perrenoud, 2001; Vacher, 2011). Les enseignants considèrent leur appropriation de l'approche en termes de rapport temps/rentabilité sur les apprentissages et de résultats aux examens. Cette réflexion sur et à la suite de l'action permet une reconceptualisation des enseignements, à la lumière des constats (Derobertmasure et al., 2010) : 
Après plusieurs années à enseigner ce module, je m’aperçois que ce premier chapitre du module me cause encore et toujours les mêmes difficultés : les élèves cherchent impérativement à apprendre par cour où se trouvent les différentes options [...]. Je répète inlassablement que je ne veux pas qu'ils les apprennent par cour [...], mais que je veux quils explorent les options et y trouvent une logique [...]. Quelques jours avant de commencer ce module avec le groupe, je revisite le PowerPoint sur la LRL et comprends que, dans le cas de ces compétences, je veux que les élèves développent leurs stratégies de Recherche des options et d'Évaluation des résultats. À partir de ce constat, je comprends que l'activité que je veux les amener à faire ce n'est pas de lire correctement les consignes écrites du cahier $[. .$.$] , mais bien à appliquer les stratégies de lecture en ligne du logiciel.$

Sa réflexion sur l'action amène cette même enseignante à ajuster, voire à reconceptualiser les tâches (Schön, 1983, 1987) et à élaborer de nouvelles façons plus efficaces d'enseigner des contenus :

Je conçois alors cet exercice en me basant sur ce qui est demandé dans les consignes écrites papier dans leur cabier, mais en les transformant en énoncés de besoin [...] cet exercice m’a pris beaucoup plus de temps en classe que normalement [...]. Il est tout de même indéniable que les élèves ont mieux compris [...].

Cette autre enseignante se questionne sur l'impact de ses actions sur tous ses élèves et dit qu'elle se réajuster en en tenant compte. Elle termine sa réflexion sur les actions menées et leurs résultats par un réajustement nécessaire pour la fois prochaine et donc une reconceptualisation de l'action à la lumière du cycle action-réflexion (Boud, 2001; Schön, 1987) :

J'ai choisi un texte trop long, plusieurs élèves semblent avoir décroché lors de l'exercice individuel. Ils l'ont bien amorcé, mais ils ont oublié la directive de noter les mots vers la fin du texte. [...] Mais c'est positif d'être capable d'apporter toutes les clarifications nécessaires à la juste compréhension. Il faut la prochaine fois choisir un texte plus court [...] Donc des exercices plus courts et plus fréquents pour développer leurs réflexes de lire de façon plus analytique.

\section{Conclusion}

Lobjectif de cet article était de décrire l'intégration déclarée, dans le journal de bord des enseignants, de stratégies de compréhension en lecture dans les cours de FP ainsi que leur enseignement inspiré de l'approche RA. Il visait aussi à décrire les retombées perçues du recours à ces stratégies pour l'enseignement d'un métier.

Comme le soulignent les recherches consultées, la pratique réflexive à l'aide du journal de bord permet à l'enseignant de se distancier des cours donnés, d'effectuer un retour sur l'action et d'ajuster ses enseignements à la lumière des retombées de l'action (enseignement) auprès des élèves. Comme le montrent les résultats présentés dans cet article, le fait de consigner régulièrement les évènements relatifs au cours offre l'opportunité de mieux adapter les enseignements aux élèves et de répondre au plus près à leurs questionnements tout en prenant en compte leurs difficultés en lecture relativement aux domaines enseignés. En outre, le suivi par journal de bord réflexif a permis de constater que les enseignants se sont approprié l'approche en l'adaptant à chacune des disciplines enseignées et aux exigences des textes à lire dans ces dernières, notamment en ce qui concerne le vocabulaire spécialisé. Le journal a aussi aidé les enseignants à réaliser l'importance de la prise en charge de la dimension 
métacognitive de l'apprentissage par les élèves et à mieux la prendre en compte par le biais, entre autres, de modelages fréquents, d'interactions régulières (en petits et grands groupes) et par la sollicitation de la réflexion à voix haute et de l'annotation. Enfin, on constate que les enseignants ont acquis de l'aisance dans la pratique guidée adaptée aux élèves et qu'ils ont mis en place des séquences d'enseignement de la compréhension de lecture qui visent l'autonomisation de leurs élèves en lecture par désétayage progressif en intégrant ces séquences à leurs propres cours et progressions, parce qu'ils en ont constaté les effets bénéfiques tant pour leur pratique que pour les apprentissages des élèves.

\section{Références}

Argyris, C. (1980). Inner contradictions of rigorous research. New York, NY : Academic Press.

Bouchard, L. (2011). Les perceptions d'étudiants au baccalauréat en sciences infirmières de l'utilisation du journal d'apprentissage (Mémoire de maitrise, Université de Montréal). Repéré à https:/papyrus.bib.umontreal.ca/xmlui/handle/1866/7076

Boud, D. (2001). Using journal writing to enhance reflective practice. New Directions for Adult and Continuing Education, (90), 9-18. http://dx.doi.org/10.1002/ace.16

Boutin, G. (2006). L'entretien de recherche qualitatif(2éd.). Sainte-Foy, QC : Presses de l'Université du Québec.

Buehl, D. (2011). Developing readers in the academic disciplines. Newark, DE : International Reading Association.

Creswell, J. W. (2012). Qualitative inquiry and research design: choosing among five approaches. Thousand Oaks : Sage.

Derobertmasure, A., Dehon, A. et Demeuse, M. (2010). Indicateurs de réflexivité en formation initiale. Analyse de contenu de dossiers réflexifs. Éducation et Formations, 1-10. Repéré à https://hal.archives-ouvertes.fr/hal-00730959/document

Deschenaux, F. (2007). La formation professionnelle au secondaire : une voie de relégation sur le marché de l'emploi?. Observatoire jeunes et société, 6(4), 1-2.

Dubeau, A., Plante, I. et Frenay, M. (2017). Achievement profiles of students in high school vocational training programs. Vocations and learning, 10, 101-120. http://dx.doi.org/10.1007/s12186-016-9163-6

Fisher, D et Frey, N. (2013). Better learning through structured teaching: A framework for the gradual release of responsibility (2 éd.). Alexandria, VA : ASCD.

Gouvernement du Québec. (2017). Régime pédagogique de la formation professionnelle (Chapitre I-13.3, r. 10). Loi sur l'instruction publique. (Chapitre I-13.3, a. 448).

Gouvernement du Québec. (2019). Programmes d'études professionnelles. Repéré à http://www.education.gouv.qc.ca/employeurs/programmes-detudes/programmes-detudes-professionnelles/

Grossmann, S., Roiné, C. et Chatigny, C. (2014). Horizons, seuils et passages. L'orientation, l'accès et le maintien des élèves en formation professionnelle à Montréal. Synthèse du rapport de recherche remis à Éducation Montréal. Repéré à https://archipel.uqam.ca/6487/1/Rapport\%20E\%CC\%81ducation\%20Montre\%CC\%81al\%20version\%20courte.pdf

Hamelin, P. (2014). Réalisation du portrait global, authentique et pratique de la formation professionnelle actuelle et récente au Québec (Mémoire de maitrise, Université du Québec à Montréal). Repéré à https://archipel.uqam.ca/6568/1/M13518.pdf

Mazalon, É. et Bourdon, S. (2015). Les choix scolaires et les obstacles à la participation des jeunes adultes non diplômés inscrits en formation professionnelle. Dans C. Villemagne et J. Myre-Bisaillon (dir.), Les jeunes adultes faiblement scolarisés. Parcours de formation et besoins d'accompagnement (p. 165-184). Québec, QC : Presses de l'Université du Québec. http://dx.doi.org/10.2307/j.ctt1f1170f.13

Meirieu, P. (s. d.). Transfert. Dans Petit dictionnaire de pédagogie. Repéré à http://www.meirieu.com/DICTIONNAIRE/dictionnaireliste.htm 
Misiorowska, M. (2014). Formation professionnelle et jeunes et adultes issus de l'immigration : état de la situation et étude exploratoire (Rapport de recherche). Montréal, QC : Commission scolaire Marguerite-Bourgeoys, Groupe de recherche immigration, équité, scolarité (GRIÉS).

Paillé, P. et Mucchielli, A. (2012). L'analyse qualitative en sciences humaines et sociales (3éd.). Paris : Armand Colin.

Perrenoud, P. (2001). Développer la pratique réflexive dans le métier d'enseignant: professionnalisation et raison pédagogique. Paris : ESF.

Pouliot, D. (2015). Mais pourquoi enseigner la lecture en FGA et en FP? Coup d'ceil sur la formation de base. Repéré à http://treaqfp.qc.ca/prive/pdf/Mais pourquoi enseigner lecture DPouliot final.pdf

Prud'homme, L. (2007). La différenciation pédagogique : analyse du sens construit par des enseignantes et un chercheur-formateur dans un contexte de recherche-action-formation (Thèse de doctorat, Université du Québec en Outaouais). Repéré à https://archipel.uqam.ca/745/1/D1599.pdf

Prud'homme, L., Dolbec, A. et Guay, M.-H. (2011). Le sens construit autour de la différenciation pédagogique dans le cadre d'une recherche-action-formation. Éducation et francophonie, 39(2), 165-188.

http://dx.doi.org/10.7202/1007733ar

Saint-Arnaud, Y. (2001). La réflexion-dans-l'action : un changement de paradigme. Recherche et formation, (36), 17-27. http://dx.doi.org/10.3406/refor.2001.1688

Saint-Arnaud, Y. (2004). L'atelier de praxéologie. Dans L. Mandeville (dir.), Apprendre autrement : pourquoi et comment (p. 103-121). Montréal, QC : Presses de l'Université du Québec.

Schön, D. A. (1983). The reflective practitioner: how professionals think in action. New York, NY : Basic Books.

Schön, D. A. (1987). Educating the reflective practitioner. San Francisco, CA : Jossey Bass.

Schoenbach, R. et Greenleaf, C. (2017). Leading for literacy: Engaging schools and districts in transforming subject-area literacy. Phi Delta Kapan, 99(3), 59-64. http://dx.doi.org/10.1177/0031721717739596

Schoenbach, R., Greenleaf, C. et Murphy, L. (2012). Reading for understanding: How reading apprenticeship improves disciplinary learning in secondary and college classrooms ( $2^{\mathrm{e}}$ éd.). San Francisco, CA : Jossey-Bass.

Shanahan, T. et Shanahan, C. (2008). Teaching disciplinary literacy to adolescents: rethinking content-area literacy. Harvard Educational Review, 78(1), 40-59. http://dx.doi.org/10.17763/haer.78.1.v62444321p602101

Statistique Canada. (2013). Les compétences au Canada : Premiers résultats du Programme pour l'évaluation internationale des compétences des adultes. Repéré à https://www150.statcan.gc.ca/n1/pub/89-555-x/89-555-x2013001-fra.htm

Tremblay, L. (2010). Modèle d'intervention pédagogique élaboré dans le but de favoriser une bonne estime de soi chez l'élève adulte de la formation professionnelle (Mémoire de maitrise, Université du Québec à Chicoutimi). Repéré à https://constellation.uqac.ca/265/1/030147865.pdf

Université de Sherbrooke. (2019). Baccalauréat en enseignement professionnel. Repéré à https://www.usherbrooke.ca/ pedagogie/programmes/1er-cycle/bep-baccalaureat-en-enseignement-professionnel/

Université du Québec à Montréal (UQAM). (2019). Baccalauréat d’enseignement en formation professionnelle et technique. Repéré à https://etudier.uqam.ca/programme? code=7414

Vacher, Y. (2011). La pratique réflexive. Recherche et formation, (66), 65-78. http://dx.doi.org/10.4000/rechercheformation.1133

Vygotsky, L. (1978). Interaction between learning and development. Dans Mind in society (p. 79-91). Cambridge, MA : Harvard University Press.

\section{Pour citer cet article}

Ouellet, C., Boultif, A. et Dubeau, A. (2020). Le journal de bord comme outil d'accompagnement d'enseignants en formation professionnelle intégrant une approche favorisant la compréhension en lecture. Formation et profession, 28(1), 69-80. http://dx.doi.org/10.18162/fp.2020.520 